\title{
Identification des propriétés mécaniques des surfaces tribologiquement transformées (TTS) à partir des essais de nano-indentation et micro-compression de piliers
}

\author{
D. Tumbajoy-Spinel ${ }^{1}$, G. Kermouche ${ }^{1}$, S. Descartes ${ }^{2}$, J.-M. Bergheau ${ }^{3}$, \\ V. Lacaille ${ }^{4,1,3}$, G. Guillonneau ${ }^{5}$ et J. Michler ${ }^{5}$
}

Reçu le 6 février 2015, accepté le 20 avril 2015

\begin{abstract}
Résumé - Les traitements mécaniques de surfaces habituellement utilisés dans l'industrie reposent sur des charges mécaniques répétées, entraînant une forte déformation plastique pouvant potentiellement induire une évolution de la microstructure proche de la surface (Transformations Tribologiques Superficielles TTS). Le gradient de taille de grains engendré permet ainsi d'améliorer les propriétés tribologiques des matériaux, ainsi que la résistance à la fatigue, l'usure et le frottement, en lien avec une amélioration des propriétés mécaniques telles que la dureté. Actuellement, un simple essai de micro-dureté n'est pas suffisant pour décrire et quantifier ces gradients. L'objet de cet article est de mettre en évidence une méthodologie de mesure du comportement élastoplastique de ces zones hyper-déformées par l'utilisation combinée d'essais d'indentation instrumentée et de compression de micro-piliers sur un matériau métallique modèle traité mécaniquement.
\end{abstract}

Mots clés : Traitement mécanique de surface / transformation tribologique superficielle (TTS) / gradient de propriétés mécaniques / nano-indentation / micro-compression de piliers

\begin{abstract}
Nano-indentation and micro-compression tests on the measurement of mechanical properties in tribologically transformed surfaces (TTS). In the industry there are several techniques which allow increasing the mechanical properties in the near-surface of materials, such as the mechanical surface treatments. In these kinds of procedures, the material is submitted to repeated mechanical loadings, producing a severe plastic deformation in the near-surface, and then leading to a local refinement of the microstructure into the affected zone (Tribologically Transformed Surfaces - TTS). This microstructure's transformation, characterized by a progressive increment of the grain size, induces a gradient of mechanical properties and better tribological properties over a few tens of microns. The main issue of this paper is to assess and describe precisely the elastic-plastic behavior of hyper-deformed surfaces in a model material, using a methodology based on nano-indentation and in-situ micro-pillars compression tests.
\end{abstract}

Key words: Mechanical surface treatments / tribologically transformed surfaces (TTS) / gradient of mechanical properties / nano-indentation / in-situ micro-compression testing

\section{Introduction}

La notion de traitement de surface est généralement associée à la modification des propriétés d'une surface par des actions d'origine physique, chimique, thermique ou métallurgique (trempe, nitruration, ... ). Dans le cas où le traitement ne repose que sur des actions mécaniques, nous pouvons parler de traitement mécanique des surfaces.

\footnotetext{
1 École des Mines de Saint-Étienne, LGF UMR 5307 CNRS, Saint-Étienne, France david.tumbajoy@emse.fr

2 Université de Lyon, CNRS, INSA-Lyon, LaMCoS, UMR 5259, 69621 Villeurbanne, France

3 Université de Lyon, ENISE, LTDS, UMR 5513 CNRS, 42023 Saint-Étienne, France

4 Winoa, 528 avenue de Savoie, BP 3, 38570 Le Cheylas, France

5 EMPA, Swiss Federal Laboratories for Materials Science and Technology, Laboratory for Mechanics of Materials and Nanostructures, Feuerwerkerstr. 39, 3602 Thun, Switzerland
} 


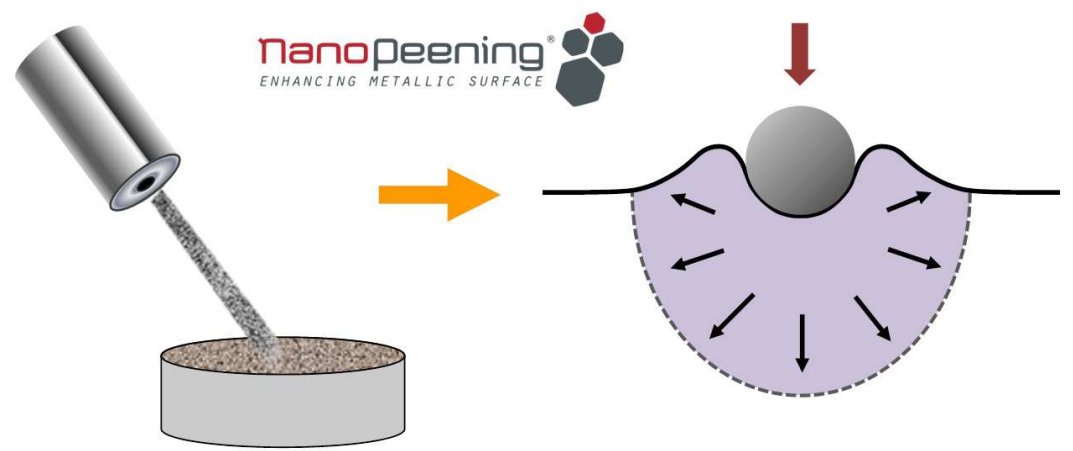

Fig. 1. Principe du traitement mécanique de surface par grenaillage intensif : Nano-Peening ${ }^{\circledR}$.

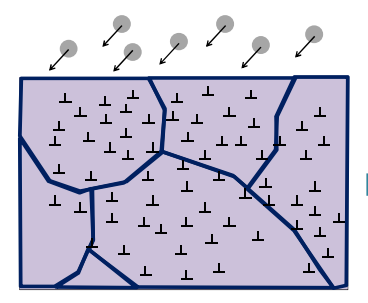

(a)

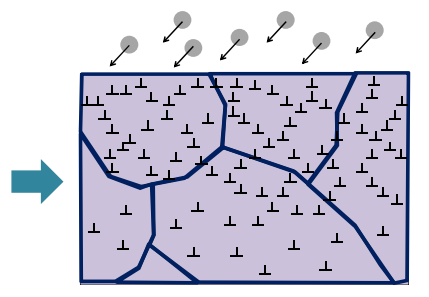

(b)

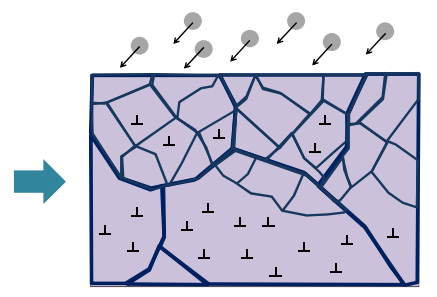

(c)

Fig. 2. Durcissement de la surface par crouissage et augmentation du nombre de joints de grains : (a) état initial de la microstructure, (b) mouvement de dislocations, (c) formation de nouveaux grains.

Les procédés les plus emblématiques sont sans conteste le grenaillage ou encore le galetage qui sont connus pour améliorer significativement la résistance à la fatigue l'introduction en sous-surface de contraintes résiduelles de compression [1-4]. Lorsque les contacts sont suffisamment sévères et répétés, ces traitements peuvent aussi engendrer des modifications microstructurales significatives en sous-surface, comme un raffinement de la taille de grain ou un hyper-écrouissage, résultant en une augmentation de la dureté locale et donc une meilleure résistance des surfaces à l'usure abrasive $[5,6]$ Ce phénomène peut être expliqué à partir des lois de Taylor et de Hall-Petch qui décrivent respectivement l'augmentation de la contrainte d'écoulement par l'augmentation de la densité de dislocation et la diminution de la taille des grains [7-9].

Les procédés « surface mechanical attrition treatment $\gg\left(\right.$ SMAT) $[6]$ et le Nano-Peening ${ }^{\circledR}[10-13]$ font partie des procédés qui ont été développés dans ce but. La zone où se produit le raffinement de taille des grains est aussi connue dans la littérature sous le nom de transformations tribologiques superficielles (TTS) $[14,15]$. Elle est caractérisée par une évolution progressive de la taille de grains avec la profondeur en sous-surface.

L'explication physique de ces évolutions microstructurales est encore aujourd'hui un verrou scientifique en raison de l'aspect multi-échelle inhérent à ce type de procédé. Les chargements sont très mal connus car de nature statistique ce qui rend difficile la compréhension du lien entre le chemin de déformation et raffinement de la taille de grain. Il convient donc aujourd'hui de développer des méthodes de caractérisations qui permettent de quantifier le gradient de propriétés mécaniques induit afin de mieux faire le lien entre chargement macroscopique et évolution mi- crostructurale. L'objet de cet article est de statuer sur la potentialité de combiner les caractérisations microstructurales (EBSD) et mécaniques (nano-indentation, nanocompression) pour quantifier ces gradients.

Pour cela il a été choisi de travailler sur les conséquences induites par le procédé de NanoPeening ${ }^{\circledR} \quad[10,13]$ qui est aujourd'hui utilisé industriellement (voir Fig. 1).

Le fer pur a été sélectionné comme matériau modèle, en raison de sa forte énergie de défaut d'empilement. À l'échelle locale, la fragmentation des grains de ce type de matériau est souvent vue comme une conséquence de la formation de murs de dislocations évoluant ensuite sous la forme de sous-joints puis de joints de grain [16], comme cela est représenté sur la Figure 2.

Dans cet article, le traitement mécanique de surface réalisé sera tout d'abord décrit ainsi que les matériaux et méthodes de caractérisation utilisées. Ensuite, les résultats des tests de nano-indentation et microcompression seront présentés et discutés. Une conclusion sera ensuite apportée sur l'intérêt de la méthodologie développée et les futurs travaux en lien.

\section{Materiau et methodes}

\subsection{Principe du Nano-Peening ${ }^{\circledR}$}

Le principe du Nano-Peening ${ }^{\circledR}$, mis au point et breveté par la société Winoa, consiste à faire une projection à haute vitesse de billes métalliques sur une surface. Plusieurs paramètres, tels que la nature des billes, la vitesse de projection, le temps d'exposition ou encore 


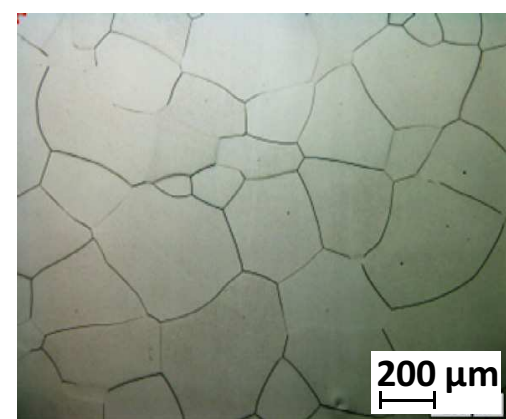

Fig. 3. Microstructure initiale du fer pur (15 ppm de carbone).

l'angle d'impact, peuvent être contrôlés (Fig. 1) [11,12]. Lors des tests réalisés en collaboration avec Winoa, les grenailles sont projetées à des vitesses comprises entre 40 et $100 \mathrm{~mm} / \mathrm{s}$ avec un angle de projection compris entre $10^{\circ}$ et $45^{\circ}$. Les billes utilisées sont fabriquées en acier traité et elles possèdent des diamètres compris entre 0.1 et $2 \mathrm{~mm}[11,12]$. Un taux de recouvrement de $5000 \%$ a été employé.

\subsection{Matériau}

Les essais ont été réalisés sur un fer quasi-pur élaboré à l'École des Mines de Saint-Étienne par une technique de fusion en creuset froid permettant de maitriser la quantité de carbone (inférieure à $15 \mathrm{ppm}$ ici). Après un fort écrouissage du matériau dans une machine à retreindre (taux de corroyage $=1,96$ ) suivi d'un recuit à $650{ }^{\circ} \mathrm{C}$ pendant $60 \mathrm{~min}$, la microstructure obtenue est caractérisée par une taille moyenne des grains de $250 \mu \mathrm{m}$ (voir Fig. 3). Ce matériau a été choisi car il possède une microstructure initiale très homogène, il ne présente pas d'inclusions et il est composé d'une seule phase (ferrite), ce qui est avantageux pour observer les transformations microstructurales générées en extrême surface. Avant le traitement mécanique du Nano-Peening ${ }^{\circledR}$, les surfaces d'intérêt sont préparées par polissage mécanique avec divers papiers abrasifs et feutres de $3 \mu \mathrm{m}$ et $1 \mu \mathrm{m}$.

\subsection{Méthodes de caractérisation de la surface transformée}

Après le traitement de surface, les échantillons sont découpés en deux afin d'observer la section transversale et ainsi pouvoir préciser le gradient de taille des grains et l'évolution des propriétés mécaniques en profondeur. La caractérisation de la sous-surface transformée est faite, pour une même zone d'intérêt :

- par microscopie électronique à balayage (MEB) Zeiss Supra 55 VP en contraste de canalisation, avec une tension de $20 \mathrm{kV}$.

- par cartographies par diffraction des électrons rétrodiffusés (EBSD) dans un MEB JEOL JSM$6500 \mathrm{~F}$, à une tension de $20 \mathrm{kV}$, en utilisant un pas d'indexation de 0,3 $\mu \mathrm{m}$. Ce pas d'acquisition a été choisi afin d'éviter des problèmes de dérive et en même temps pouvoir garantir un temps d'acquisition raisonnable (30 h environ). La détection des grains avec une taille inferieure à $300 \mathrm{~nm}$ reste très réduite.

Pour chacune des analyses, la surface a été préparée avec un polissage mécanique (papiers abrasifs et feutres de $3 \mu \mathrm{m}$ et $1 \mu \mathrm{m}$ ) et une finition à la silice collö̈dale. La Figure 4 présente la section transversale d'une zone traitée par Nano-Peening ${ }^{\circledR}$.

\subsection{Fabrication de micro-piliers dans la section transversale de l'échantillon}

Afin d'estimer la variation de propriétés mécaniques engendrée dans la zone TTS (Fig. 4), deux micro-piliers ont été usinés par faisceau ionique dans un microscope double faisceau (FIB ZEISS NVision40) en 4 étapes successives afin d'éviter le ré-dépôt du matériau abradé par le faisceau d'ions [17-19]. À chaque étape la valeur du courant ionique est diminuée afin de produire des surfaces de plus en plus homogènes et lisses [20-22]. La tension du faisceau d'ions de Galium $\left(\mathrm{Ga}^{+}\right)$est de $30 \mathrm{kV}$ avec une gamme de courant de $27 \mathrm{nA}$ à $700 \mathrm{pA}$.

Les micro-piliers sont usinés en deux endroits différents de la sous-surface, un dans la zone microstructurée TTS et l'autre dans un grain de la zone non affectée par le Nano-Peening ${ }^{\circledR}$, i.e. sans transformation microstructurale. Le pilier de la zone hyper-déformée a été réalisé à une profondeur de l'ordre de $15 \mu \mathrm{m}$, où la taille moyenne des grains est de l'ordre de $800 \mathrm{~nm}$. Le diamètre du pilier est de $9.3 \mu \mathrm{m}$, sa hauteur de $\sim 24 \mu \mathrm{m}$ (ratio 2,6 ). En ce qui concerne le pilier réalisé dans un seul grain, son diamètre est de $\sim 5.4 \mu \mathrm{m}$ et sa hauteur de $\sim 10.4 \mu \mathrm{m}$ (ratio 1,92). Ces piliers possèdent une forme tronconique avec un angle d'ouverture de cône de l'ordre de $5^{\circ}$ et $9^{\circ}$, respectivement. La distribution des micro-piliers dans l'échantillon est présentée dans la Figure 5.

\subsection{Méthodes de mesure de propriétés mécaniques}

La quantification du gradient des propriétés mécaniques généré par la transformation microstructurale du matériau est réalisée avec deux techniques différentes : la nano-indentation et la micro-compression de piliers. La nano-indentation est une technique qui permet de mesurer propriétés comme la dureté ou le module d'élasticité, alors que la compression des micro-piliers conduit à l'identification des contraintes et déformations très localement. Les procédés implémentés et ses caractéristiques principales sont présentés par la suite.

\section{- Nano-indentation :}

Les données expérimentales obtenues avec un essai d'indentation standard sont souvent présentées dans une 


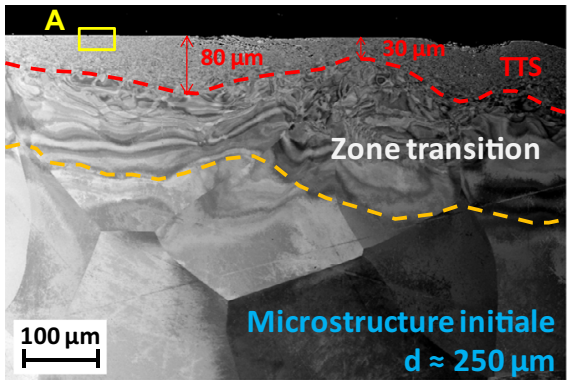

(a)

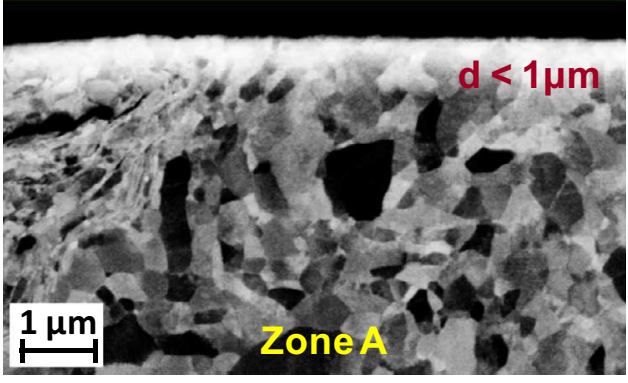

(b)

Fig. 4. Caractérisation de la microstructure transformée après traitement mécanique de surface : (a) vue globale de la soussurface, (b) TTS.
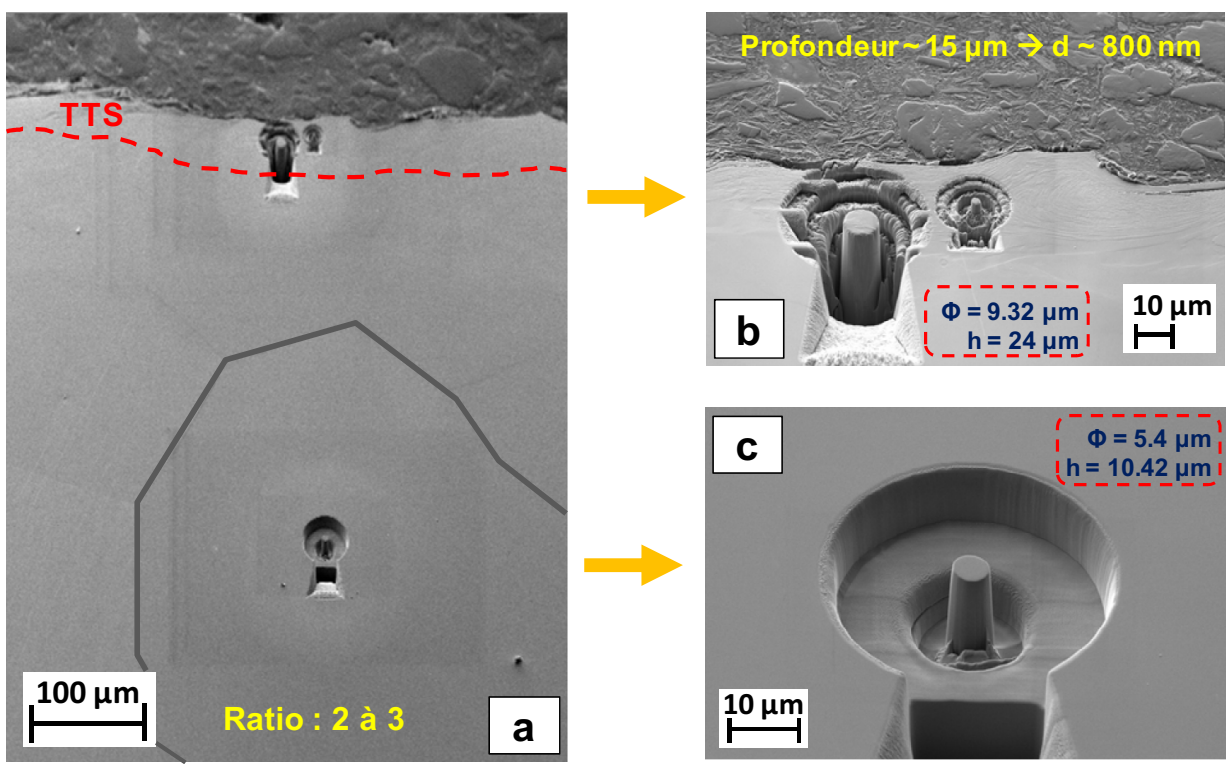

Fig. 5. (a) Distribution de Micro-piliers dans l'échantillon, (b) micro-pilier dans la zone TTS $(\phi=9.32 \mu \mathrm{m})$ et (c) micro-pilier dans la zone sans transformation microstructurale $(\phi=5.4 \mu \mathrm{m})$.

courbe qui relie la force $(F)$ et la pénétration $(h)$ de l'indenteur, tel qu'il est représenté dans le schéma de la Figure 6 . Dans le point de force maximale $\left(F_{\max }\right)$, il est possible d'estimer la raideur de contact $(S)$ lors de la décharge de l'indenteur, à partir de laquelle la hauteur et l'aire de contact peuvent être calculées [23-25]. Ces valeurs permettent de quantifier la dureté et le module d'élasticité réduit du matériau indenté à partir du point maximal de la courbe de charge et de la pente initiale de décharge [26].

200 tests d'indentation ont été effectués dans la section transversale de l'échantillon afin de cartographier les propriétés mécaniques dans la zone transformée d'intérêt et remonter au gradient de propriétés mécaniques engendré par le grenaillage. Ces indentations ont été réalisées dans la même zone repérée et caractérisée par imagerie MEB et cartographie EBSD. Les indentations ont été faites avec une machine d'indentation NHT, développée par la société CSM Instruments SA ${ }^{\circledR}$ (Suisse). Celles-ci sont illustrées dans la Figure 7.

Les 200 indentations ont été faites par l'intermédiaire d'une matrice d'empreintes $10 \times 20$ espacées de $15 \mu \mathrm{m}$ entre elles dans les directions verticales et horizontales. Ainsi 20 profondeurs différentes par rapport à l'extrême surface avec 10 essais par profondeur ont été testées. Les indentations correspondent aux essais standards avec une pointe Berkovich en diamant, à une vitesse de chargedécharge de $20 \mathrm{mN} / \mathrm{min}$ jusqu'une force maximale de $10 \mathrm{mN}$.

La Figure 7 illustre deux types d'empreintes selon la profondeur par rapport à la surface. Dans le cas des empreintes placées à $\sim 2 \mu \mathrm{m}$ de la surface, on constate une taille caractéristique de $2.8 \mu \mathrm{m}$. En revanche, dans le cas des empreintes plus écartées de la surface, à $\sim 285 \mu \mathrm{m}$ de la zone impactée, la taille caractéristique augmente vers $4 \mu \mathrm{m}$. Cette augmentation de taille de l'empreinte est cohérente avec le gradient de propriétés mécaniques induit par les variations microstructurales du matériau selon la profondeur.

Les empreintes présentent un bourrelet (Fig. 7) lié à la déformation plastique générée dans le matériau lors des essais d'indentation. Dans le cas des matériaux malléables comme le fer pur, il est pertinent de prendre une méthode de dépouillement qui considère la formation de bourrelet 


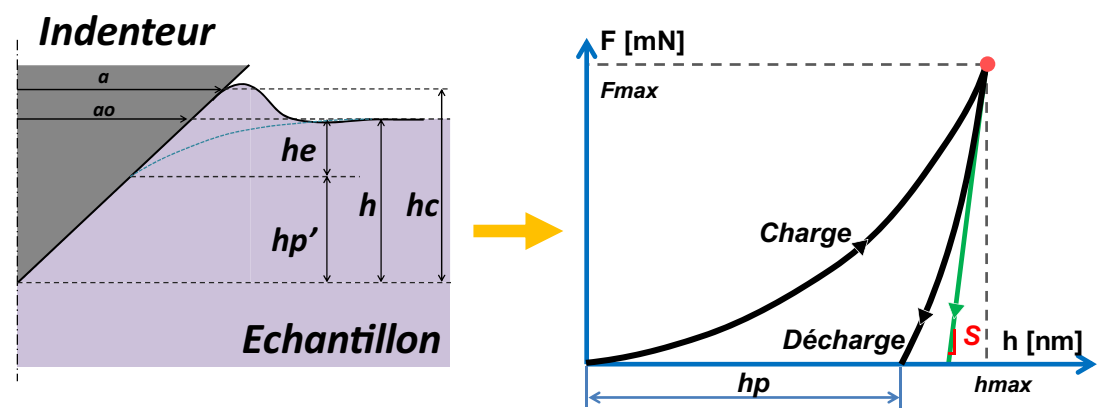

Fig. 6. Représentation caractéristique d'une courbe force - pénétration pour un test d'indentation standard.

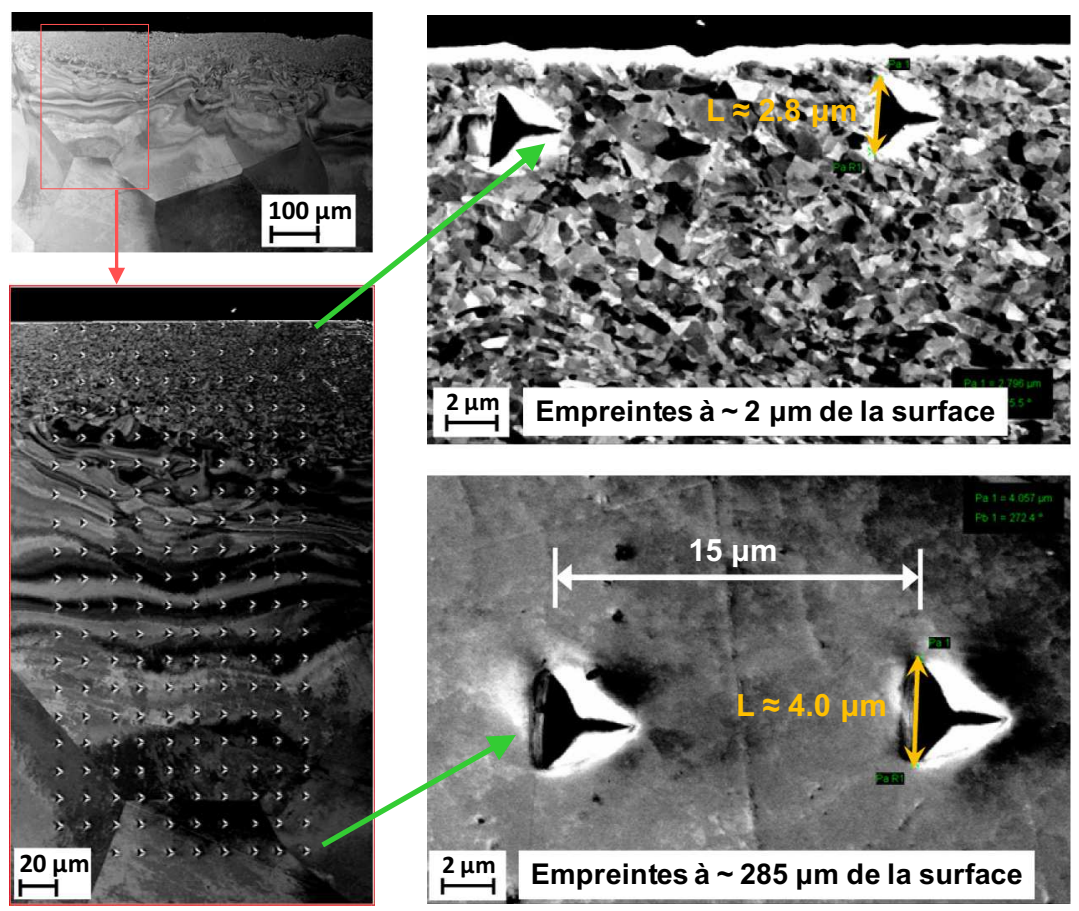

Fig. 7. Matrice d'indentations standards $(10 \times 20)$ dans la section transversale d'une surface hyper-déformée. Indentations avec une pointe Berkovich et une force de $10 \mathrm{mN}$.

afin de mieux estimer l'aire de contact projeté et par conséquent la valeur de dureté. C'est pour cette raison que la méthode de Loubet [26,27] a été choisie pour le dépouillement des données d'indentation (Fig. 6).

\section{- Micro-compression de piliers :}

Les essais de micro-compression des piliers ont été effectués avec un indenteur In Situ à l'EMPA-Thoune (Suisse) Pour les deux tests de compression un poinçon plat de $15 \mu \mathrm{m}$ de diamètre a été employé. Les résultats obtenus seront présentés dans la section suivante.

\section{Résultats}

\subsection{Microstructure après Nano-Peening ${ }^{\circledR}$}

À partir des observations MEB présentées dans la Figure 4 , trois zones bien définies sont mises en évidence (Fig. 4a). La première zone correspond à une couche submicrométrique avec une épaisseur non-homogène de l'ordre de $50 \mu \mathrm{m}$ en moyenne. Cette zone est formée à partir des grains d'une taille inferieure au micromètre (Fig. 4b). La deuxième zone correspond à une région de transition, où malgré le fort écrouissage dans le matériau, les désorientations à l'intérieur des cristaux ne sont pas suffisantes pour former des nouveaux joints des grains [28]. Finalement, la troisième zone observable correspond à l'état initial du matériau où la microstructure n'a pas été transformée et la taille moyenne de grain reste à $\sim 250 \mu \mathrm{m}$.

Afin de mieux caractériser la surface transformée et identifier une statistique de taille de grain, une cartographie EBSD a été effectuée dans la même région repérée par imagerie MEB. Une zone submicrométrique est identifiée (Fig. 8a) dans laquelle la taille de grains passe de $0.76 \mu \mathrm{m}$ jusqu'à $5.82 \mu \mathrm{m}$, respectivement en extrême surface et à une profondeur de $60 \mu \mathrm{m}$. En extrême surface, un ratio de réduction du cristal de l'ordre de 300 fois par rapport à la taille initiale de grains est mesuré. La courbe 


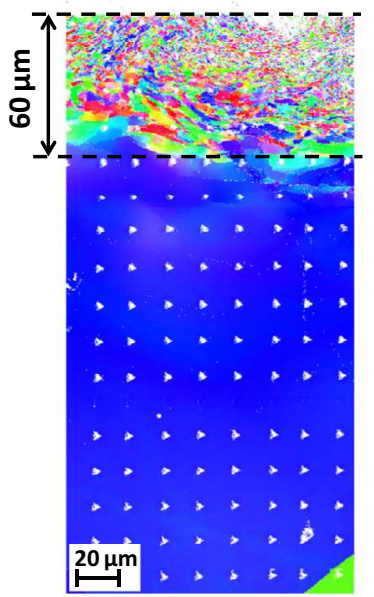

(a)

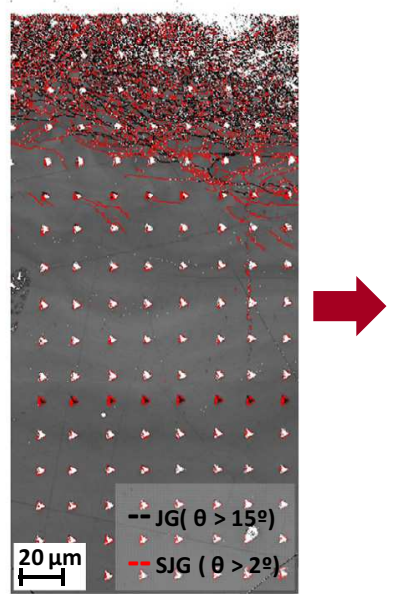

(b)

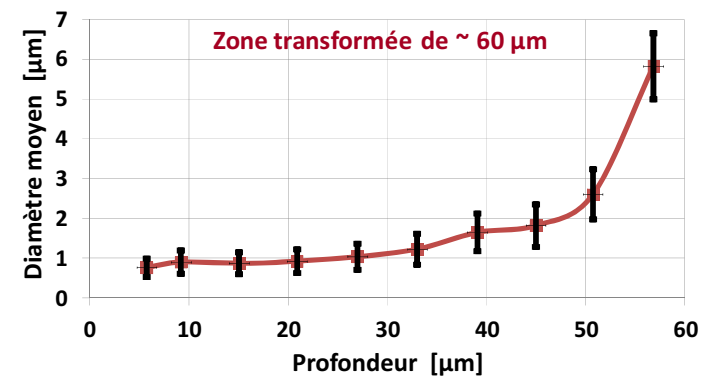

(c)

Fig. 8. Zone transformée : (a) cartographie EBSD, (b) joints et sous-joints de grains. (c) Statistiques de taille de grains en fonction de la profondeur dans la section transversale.

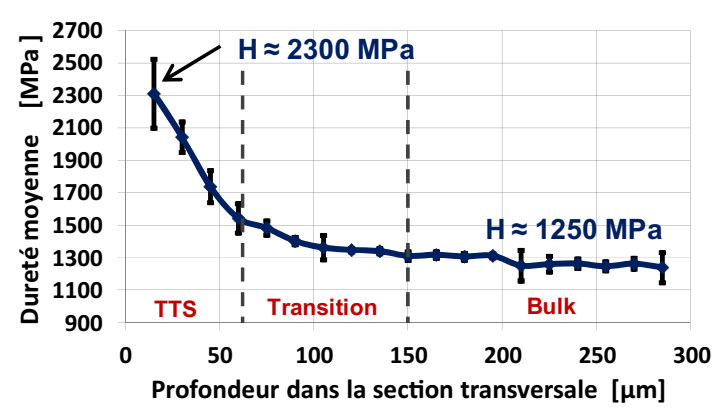

(a)

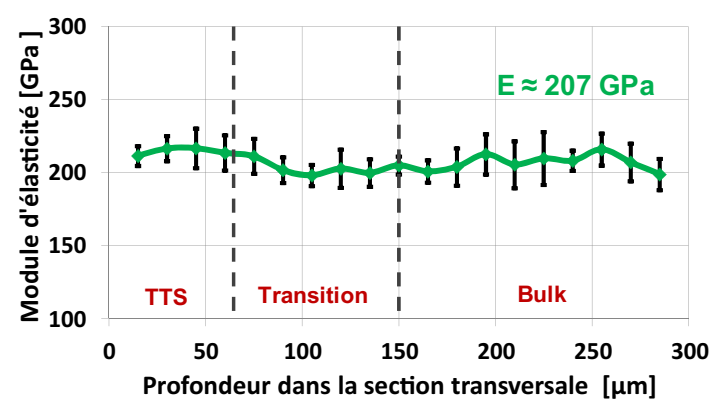

(b)

Fig. 9. Évolution de (a) la dureté moyenne [MPa] et (b) du module d'élasticité [GPa] dans la section transversale d'une zone TTS. Dépouillement des courbes avec la méthode Loubet.

de la Figure 8c décrit l'augmentation du diamètre moyen de taille de grain en fonction de la profondeur dans les $60 \mu \mathrm{m}$ d'épaisseur de la surface submicrométrique. La zone de transition est identifiée par cartographie EBSD comme une zone homogène avec de faibles désorientations cristallines.

La Figure $8 \mathrm{~b}$ présente également une cartographie des joints de grains (JG) et des sous-joints de grains (SJG) en fonction de différentes désorientations cristallines $(\theta)[28,29]$. Les grandes désorientations cristallines sont majoritairement présentes dans la zone submicrométrique, raison pour laquelle cette zone est formée de plusieurs grains de l'ordre du micromètre. En revanche, la zone de transition est composée spécialement par des sous-joints de grains [30], c'est-à-dire que cette zone ne contient pas de nouveaux grains formés, malgré la déformation plastique subie [28]. Dans cette cartographie, les traces blanches triangulaires correspondent aux empreintes d'indentations réalisées sur cette zone d'intérêt.

\subsection{Gradient de dureté par nano-indentation}

L'évolution de la dureté moyenne et module d'Young moyen en fonction de la profondeur (Fig. 9) est obtenue à partir des mesures d'indentation. Chaque point de la courbe correspond à la moyenne des valeurs trouvées à une même profondeur. Pour le cas du premier point de chaque courbe, correspondant aux indentations effectuées à $2 \mu \mathrm{m}$ de l'extrême surface, les mesures obtenues sont aberrantes et elles ne sont pas prises en compte.

Dans le profil de dureté une diminution de $2300 \mathrm{MPa}$ en extrême surface jusqu'à $1250 \mathrm{MPa}$ à une profondeur de $150 \mu \mathrm{m}$ est observée, ce qui correspond à une réduction totale de l'ordre de $40 \%$. Cette baisse de dureté est majoritairement présente dans les $60 \mu \mathrm{m}$ d'épaisseur de la zone microstructurée TTS $(30 \%)$, alors que dans la zone de transition la diminution de dureté est moins prononcée $(10 \%)$. À la fin de la zone de transition, c'està-dire à $\sim 150 \mu \mathrm{m}$ de profondeur, la dureté se stabilise. De plus le module d'élasticité est quasi-indépendant de la microstructure avec une valeur de $\sim 207$ GPa ce qui était attendu [31].

\subsection{Micro-compression}

Une séquence d'images de chaque essai de compression est illustrée dans la Figure 10. Pour le test de compression dans la zone sans transformation microstructurale 

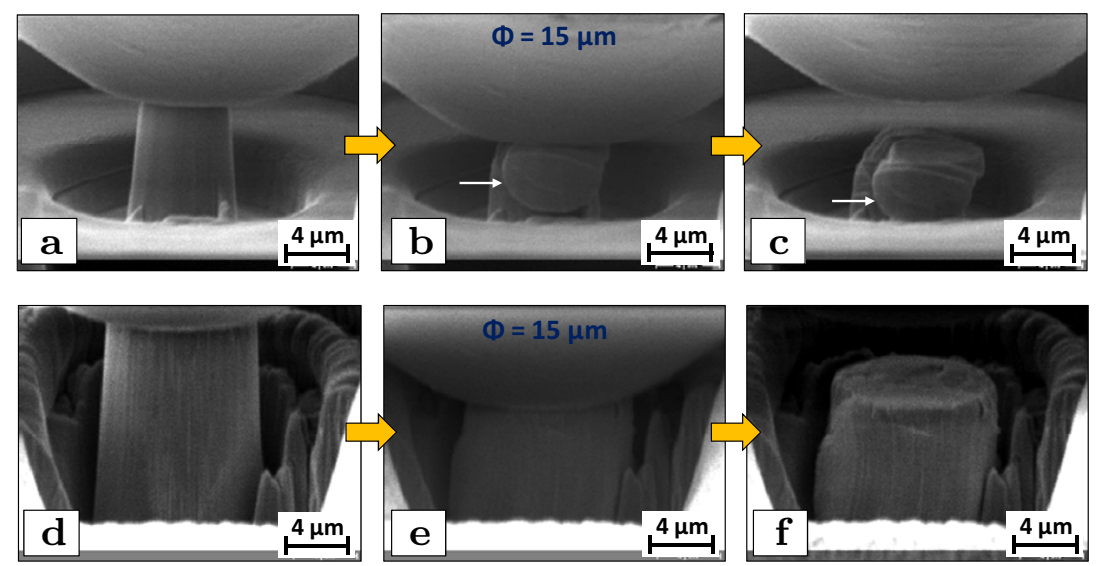

Fig. 10. Compression des piliers dans la zone sans transformation microstructurale (a, b, c) et dans la zone TTS (d, e, f) avec un poinçon plat de $\phi 15 \mu \mathrm{m}$.

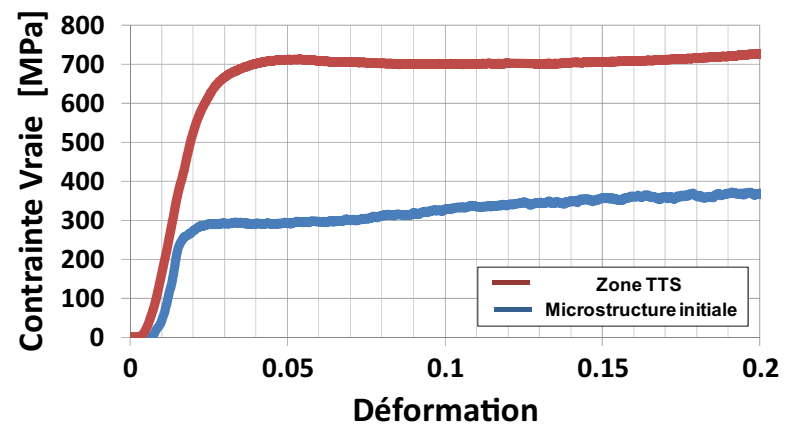

Fig. 11. Courbes des contraintes vraies et déformations pour les piliers de la zone TTS et la zone sans transformation microstructural (microstructure initiale.)

(Figs. 10a-10c), le pilier se déforme selon un plan de glissement préférentiel du grain [32] ; comme un seul grain est sollicité tel qu'il est indiqué par les flèches blanches des images 10b et 10c. En revanche, le pilier de la zone TTS (Figs. 10d-10f) se déforme de façon très homogène lors de la compression, ce qui est dû aux différentes orientations cristallines des grains qui le composent.

À partir de ces tests la courbe force - déplacement pour chaque pilier est obtenue, et permet de remonter à la courbe contrainte - déformation (Fig. 11). Dans le dépouillement de résultats l'influence du bâti, le substrat et le porte-échantillon ont été pris en compte.

\section{Discussion}

\subsection{Relation entre le gradient de dureté et la taille des grains}

Observons tout d'abord que le profil de dureté varie sur les 150 premiers microns (avec une diminution de l'ordre de $40 \%$ de la valeur de dureté) alors que la zone submicrométrique ne dépasse pas les $60 \mu \mathrm{m}$ de profondeur. Ceci s'explique par la présence d'une zone de transition entre la surface transformée et la microstructure initiale du fer pur.

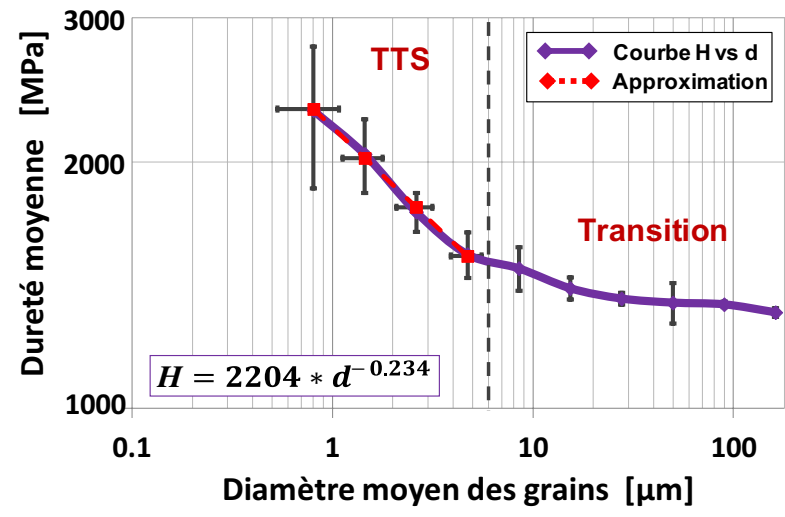

Fig. 12. Évolution de la Dureté $(\mathrm{H})$ en fonction du diamétre moyen des grains (d) dans la zone de transformation microstructurale.

Sur la courbe de la Figure 12, représentée en échelle logarithmique, il apparait clairement que la dureté moyenne diminue lorsque la taille de grain de la microstructure augmente. Une linéarité de la courbe est constaté pour une taille de grain inferieure à $\sim 6 \mu \mathrm{m}$, correspondant aux $60 \mu \mathrm{m}$ de profondeur de la zone nanostructurée TTS. Au contraire, cette tendance linéaire disparait dans la zone de transition, où la courbe correspond en réalité à une extrapolation de la statistique de taille de grain de la région submicrométrique.

La section linéaire de la courbe est décrite dans la zone TTS à partir d'une approximation correspondante à une loi puissance reliant la dureté moyenne $(H)$ en fonction de la taille de grain $(d)$. Cette expression peut être mise en relief vis-à-vis de la loi de Hall-Petch, en substituant à la dureté, la limite d'élasticité du matériau. Néanmoins l'exposant mesuré $(n=-0,23)$ est significativement différent de celui attendu avec cette loi $(n=-0,5)[8,33]$. Ce dernier aspect s'explique parce que l'approximation linéaire est faite avec la valeur absolue de la dureté, au lieu de la valeur relative qui prendrait en compte une dureté caractéristique du matériau $\left(H_{o}\right)$ [33]. Par exemple, si une valeur relative de dureté $\left(H-H_{o}\right)$ était considérée, avec 
une valeur de $H_{o}=1000 \mathrm{MPa}$, l'exposant de l'expression correspondrait à une valeur de -0.51 .

L'absence des grains bien définis dans la zone de transition ne permet pas de caractériser le gradient de propriétés mécaniques en fonction de la microstructure. En revanche, l'existence des déformations plastiques est bien identifiée dans cette zone (Fig. 4), ce qui explique la présence d'un gradient de dureté entre $60 \mu \mathrm{m}$ et $150 \mu \mathrm{m}$ de profondeur. C'est pourquoi il est possible de supposer que la diminution de dureté dans la zone de transition n'est pas seulement une conséquence de l'évolution de la taille de grains (loi de Hall-Petch), mais peut être aussi liée à une diminution de la densité de dislocations en profondeur (Loi de Taylor) [9,34]. Néanmoins, l'influence des contraintes résiduelles sur l'évolution des propriétés mécaniques en profondeur reste encore à être mise en évidence, ce qui peut donner d'autres éléments de réponse à ce sujet.

\subsection{Nano-indentation vs. nano-compression}

Nous pouvons observer sur les Figures 9 et 11 une différence significative en terme de dureté et de limite d'élasticité entre la zone TTS et le volume du matériau. Les essais de micro-compression montrent un rapport supérieur à 2 entre la limite d'élasticité du volume et celui de la zone TTS, en accord avec les résultats de nanoindentation. Par ailleurs, l'essai de micro-compression, plus riche que l'essai d'indentation, permet de mettre en lumière une absence d'écrouissage de la zone à grains fins. Ce phénomène peut s'expliquer par un écoulement plastique piloté par les joints de grains [35] et donc un faible effet de durcissement induit par un mécanisme de type Taylor. Une plus grande sensibilité à la vitesse de déformation est donc attendue et sera l'objet d'études ultérieures.

Les résultats d'indentation et micro-compression des piliers montrent une évolution de propriétés mécaniques en profondeur comme une conséquence de la transformation microstructurale des matériaux. Tabor avait proposé à partir d'une approche empirique que la limite élastique $\left(\sigma_{y}\right)$ d'un matériau métallique est relié à la dureté par un facteur d'un tiers environ [36].

En comparant les deux résultats de micro-compression avec le profil de dureté $\left(H / \sigma_{y}\right)$, la zone à grains fins (TTS) est caractérisée par un rapport de $\sim 3,3$ (2300 MPa/700 MPa), alors que pour la microstructure initiale il y a un rapport de $\sim 4,2(1250 \mathrm{MPa} / 300 \mathrm{MPa})$. Notons que la comparaison entre valeur d'indentation et de compression dans le volume doit être interprétée avec précaution comme l'orientation des grains n'est pas la même dans les deux cas. Ce facteur 4,2 peut donc trouver son origine ici. Néanmoins, les rapports de dureté et limite d'élasticité dans les deux cas semblent cohérents avec la littérature [37].

Nous précisons également qu'un potentiel effet d'échelle est considéré comme négligeable en raison de l'importante différence observée entre les courbes contrainte-déformation de la zone TTS et la zone nontransformée. De plus l'effet d'échelle est classiquement présent dans les piliers submicrométriques [19,22], ce qui n'est pas le cas ici malgré les différentes dimensions des piliers.

\section{Conclusions et perspectives}

Cet article expose deux méthodes complémentaires, indentation et micro-compression de piliers, pour identifier le gradient de propriétés mécaniques en sous-surface d'un matériau métallique, ainsi qu'un lien entre celles-ci. Pour mettre en évidence cela, un traitement mécanique de surface, le Nano-Peening ${ }^{\circledR}$, a été appliqué pour un matériau de fer pur, générant ainsi une transformation microstructurale dans la sous-surface

Dans un premier temps, il a été mis en évidence que l'indentation est une technique qui permet non-seulement de quantifier le gradient de propriétés mécaniques, mais encore de constater l'influence de la taille des grains sur celles-ci, ainsi que la compréhension des phénomènes qui gouvernent le comportement mécanique du matériau après un traitement comme le grenaillage.

Mais les résultats d'indentation sont intrinsèques à la méthode de dépouillement employée. Donc dans un deuxième temps, ceux-ci ont été confrontés aux résultats obtenus par compression de micro-piliers. La faisabilité des tests de micro-compression a tout d'abord été vérifiée dans les zones hyper-déformées et sans transformation microstructurale du fer pur. Puis les résultats de microcompression ont confirmé une augmentation des propriétés mécaniques dans la zone TTS par rapport au matériau initial (non transformé).

Ainsi une correspondance entre les résultats des deux techniques employées est mise en évidence en comparant le pourcentage d'incrément de propriétés mécaniques, ainsi que le rapport expérimental obtenu entre la dureté et limite élastique pour une même profondeur [36,37]. Il est important de signaler que l'indentation est une méthode beaucoup plus accessible du point de vue technologique par rapport à la micro-compression de piliers. En considérant la cohérence entre résultats, l'indentation reste une technique prometteuse et avantageuse dans l'identification de propriétés mécaniques lorsque le dépouillement des résultats est soigneusement réalisé. Quant à la micro-compression de piliers, cette technique offre l'avantage d'un moyen direct pour accéder à une loi constitutive locale du matériau.

En ce qui concerne le matériau des échantillons, il a été constaté que le fer pur est un matériau approprié pour produire des zones TTS exploitables face aux techniques envisagées pour mesurer le gradient de propriétés mécaniques.

En perspectives de ce travail, il est envisagé de confronter les résultats obtenus par compression de micropiliers avec les points représentatifs de contraintes et déformations calculables à travers des résultats d'un essai d'indentation conventionnel [38-40]. Il serait également 
intéressant de caractériser le gradient de propriétés des zones TTS obtenues à partir d'autres procédés permettant des très grandes déformations, tels que le procédé de torsion sous pression $[14,15,41]$ ou la micropercussion $[42,43]$.

Remerciements. Les auteurs tiennent à remercier le LABEX MANUTECH-SISE (ANR-10-LABX-0075) de l'Université de Lyon dans le cadre du programme «Investissements d'Avenir » (ANR-11-IDEX-0007) géré par l'Agence Nationale de Recherche Française (ANR). Les auteurs remercient également S. SaoJoao, M. Mondon et G. Blanc de l'École des Mines de Saint-Étienne pour leurs aides sur la préparation des échantillons et leur caractérisation microstructurale. Ils tiennent aussi à remercier le CLYM (Centre LYonnais de Microscopie, http ://clym.insa-lyon.fr) pour avoir donné accès au FIB/SEM (Zeiss NVision 40) et à N. Blanchard, A. DescampsMandine, Th. Douillard, et B. Van De Moortèle pour leur soutien technique. CLYM est soutenu par le CNRS, le Grand Lyon et le Conseil Régional Rhône-Alpes (France).

\section{Références}

[1] H.W. Zhang, Z.K. Hei, G. Liu, J. Lu, K. Lu, Acta Mater. 51 (2003) 1871-1881

[2] M. Korzynski, Int. J. Machine Tools Manuf. 47 (2007) 1956- 1964

[3] M.M. El-Khabeery, M. Fattouh, Int. J. Machine Tools Manuf. 29 (1989) 391-401

[4] G. Kermouche, Matériaux \& Techniques 101 (2013) 308

[5] K. Lu, J. Lu, Mater. Sci. Eng. A 375-377 (2004) 38-45

[6] N.R. Tao, Z.B. Wang, W.P. Tong, M.L. Sui, J. Lu, K. Lu, Acta Mater. 50 (2002) 4603-4616

[7] P. Bazarnik, Y. Huang, M. Lewandowska, T.G. Langdon, Mater. Sci. Eng. A 626 (2015) 9-15

[8] J. Aldazabal, J. Gil Sevillano, Mater. Sci. Eng. A 365 (2004) 186-190

[9] H. Parvin, M. Kazeminezhad, Comput. Mater. Sci. 95 (2014) 250-255

[10] T. Prezeau, T. Müller, E. Dransart, Y. Giraud, Traitements et Matériaux 412 (2011) 37-43

[11] T. Prezeau, T. Müller, J. Samuel, Traitement de surface d'une pièce métallique par grenaillage oblique, Brevet EP 2659010 A1

[12] T. Prezeau, T. Müller, J. Samuel, Surface treatment of a metal part, Brevet US 20140166160 A1

[13] V. Lacaille, G. Kermouche, D.Y. Tumbajoy Spinel, E. Feulvarch, C. Morel, J.M. Bergheau, IOP Conf. Ser.: Mater. Sci. Eng. 63 (2014) 012124

[14] S. Descartes, M. Busquet, Y. Berthier, Wear 271 (2011) 1833-1841.

[15] S. Descartes, C. Desrayaud, E.F. Rauch, Mater. Sci. Eng. A 528 (2011) 3666-3675.
[16] Y.H. Zhao, H.W. Sheng, K. Lu, Acta Mater. 49 (2001) 365-375

[17] M. Uchic, D.M. Dimiduk, Mater. Sci. Eng. A 400-401 (2005) 268-278

[18] M. Mutoh, T. Nagoshi, T.F. Mark-Chang, T. Sato, M. Sone, Microelectronic Engineering 111 (2013) 118-121

[19] J.R. Greer, W.C. Oliver, W.D. Nix, Acta Mater. 53 (2005) 1821-1830

[20] G. Monnet, M.A. Pouchon, Mater. Lett. 98 (2013) 128130

[21] E.M. Grieveson, D.E.J. Armstrong, S. Xu, S.G. Roberts, J. Nucl. Mater. 430 (2012) 119-124

[22] C.P. Frick, B.G. Clark, S. Orso, A.S. Schneider, E. Arzt, Mater. Sci. Eng. A 489 (2008) 319-329

[23] G. Kermouche, J.L. Loubet, J.M. Bergheau, Mech. Mater. 40 (2008) 271-283

[24] J. Woirgard, J.C. Dargenton, C. Tromas, V. Audurier, Surf. Coat. Technol. 100-101 (1998) 103-109

[25] J.L. Bucaille, S. Stauss, E. Felder, J. Michler, Acta Materialia 51 (2003) 1663-1678

[26] G. Guillonneau, G. Kermouche, S. Bec, J.L. Loubet, Tribol. Int. 70 (2014) 190-198

[27] J.L. Loubet, M. Bauer, A. Tonck, S. Bec, B. GauthierManuel, Nano-indentation with a surface force apparatus, NATO Advanced Study Institute Series E (1993) 429-447

[28] Yu. Ivanisenko, R.Z. Valiev, H.J. Fecht, Mater. Sci. Eng. A 390 (2005) 159-165

[29] M. El Wahabi, L. Gavard, J.M. Cabrera, J.M. Prado, F. Montheillet, Mater. Sci. Eng. A 393 (2005) 83-90

[30] S. Gourdet, F. Montheillet, Mater. Sci. Eng. A 283 (2000) 274-288

[31] G.E. Fougere, L. Riester, M. Ferber, J.R. Weertman, R.W. Siegel, Mater. Sci. Eng. A 204 (1995) 1-6

[32] B.R. Sørås Rogne, C. Thaulow, Mater. Sci. Eng. A 621 (2015) 133-142

[33] K.A. Padmanabhan, G.P. Dinda, H. Hahn, H. Gleiter, Mater. Sci. Eng. A 452-453 (2007) 462-468

[34] D. Kuhlmann-Wilsdorf, Mater. Sci. Eng. A 113 (1989) $1-41$

[35] Y. Champion, Mater. Sci. Eng. A 560 (2013) 315-320

[36] I. Brooks, P. Lin, G. Palumbo, G.D. Hibbard, U. Erb, Mater. Sci. Eng. A 491 (2008) 412-419

[37] P. Zhang, S.X. Li, Z.F. Zhang, Mater. Sci. Eng. A 529 (2011) 62-73

[38] G. Kermouche, A.L. Kaiser, P. Gilles, J.M. Bergheau, Wear 263 (2007) 1551-1555

[39] M. Dao, N. Chollacoop, K.J. Van Vliet, T.A. Venkatesh, S. Suresh, Acta Mater. 49 (2001) 3899-3918

[40] J.M. Collin, G. Mauvoisin, O. Bartier, R. El Abdi, P. Pilvin, Mater. Sci. Eng. A 501 (2009) 140-145

[41] A. Loucif, R.B. Figueiredo, T. Baudin, F. Brisset, R. Chemam, T.G. Langdon, Mater. Sci. Eng. A 532 (2012) $139-145$

[42] G. Kermouche, G. Pacquaut, C. Langlade, J.M. Bergheau, C. R. Mécanique 339 (2011) 552-562

[43] H. Al Baida, C. Langlade, G. Kermouche, R. Ambriz, Matériaux \& Techniques 102 (2014) 604 